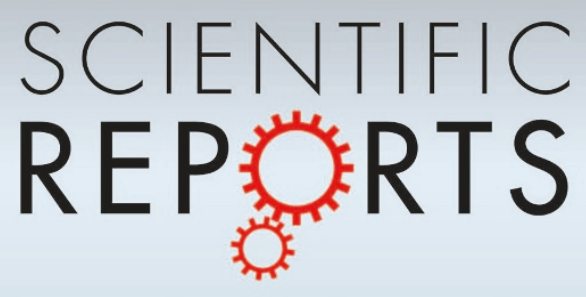

OPEN

SUBJECT AREAS:

NANOSCIENCE AND

TECHNOLOGY

MATERIALS SCIENCE

TWO-DIMENSIONAL MATERIALS

Received

17 February 2014

Accepted

12 June 2014

Published

30 June 2014

Correspondence and requests for materials should be addressed to K.X. (xiaok@ornl.gov)

\section{Controlled Vapor Phase Growth of Single Crystalline, Two-Dimensional GaSe Crystals with High Photoresponse}

Xufan Li, Ming-Wei Lin, Alexander A. Puretzky, Juan C. Idrobo, Cheng Ma, Miaofang Chi, Mina Yoon, Christopher M. Rouleau, Ivan I. Kravchenko, David B. Geohegan \& Kai Xiao

Center for Nanophase Materials Sciences, Oak Ridge National Laboratory, One Bethel Valley Road, Oak Ridge, TN, 37831 , USA.

Compared with their bulk counterparts, atomically thin two-dimensional (2D) crystals exhibit new physical properties, and have the potential to enable next-generation electronic and optoelectronic devices. However, controlled synthesis of large uniform monolayer and multi-layer $2 \mathrm{D}$ crystals is still challenging. Here, we report the controlled synthesis of $2 \mathrm{D} \mathrm{GaSe}$ crystals on $\mathrm{SiO}_{2} / \mathrm{Si}$ substrates using a vapor phase deposition method. For the first time, uniform, large (up to $\sim 60 \mu \mathrm{m}$ in lateral size), single-crystalline, triangular monolayer GaSe crystals were obtained and their structure and orientation were characterized from atomic scale to micrometer scale. The size, density, shape, thickness, and uniformity of the 2D GaSe crystals were shown to be controllable by growth duration, growth region, growth temperature, and argon carrier gas flow rate. The theoretical modeling of the electronic structure and Raman spectroscopy demonstrate a direct-to-indirect bandgap transition and progressive confinement-induced bandgap shifts for 2D GaSe crystals. The 2D GaSe crystals show p-type semiconductor characteristics and high photoresponsivity $(\sim 1.7 \mathrm{~A} / \mathrm{W}$ under white light illumination) comparable to exfoliated GaSe nanosheets. These $2 \mathrm{D}$ GaSe crystals are potentially useful for next-generation electronic and optoelectronic devices such as photodetectors and field-effect transistors.

T wo-dimensional (2D) nanomaterials with single or few atomic layers exhibit many unique physical and chemical properties when compared to their bulk counterparts ${ }^{1-4}$. This is easily seen in the case of graphene, which exhibits exotic physical properties never observed from bulk graphite ${ }^{2,5-7}$. The controlled synthesis and processing of graphene has opened up new avenues to study the role of dimensionality on the fundamental properties of materials, and has triggered the development of synthesis approaches for other $2 \mathrm{D}$ materials ${ }^{4}$. Although graphene shows excellent electrical properties, e.g., high electron and hole mobility $\left(\sim 10^{6} \mathrm{~cm}^{2} / \mathrm{Vs}\right)$, its zero bandgap energy limits its applications in logic electronics and field-effect transistors (FETs) ${ }^{4,7}$. In order to overcome these limitations, a variety of 2D materials beyond graphene with different bandgaps have been synthesized in recent years ${ }^{4,8}$, including insulating $h$ - $\mathrm{BN}^{9,10}$ and semiconducting layered transition metal dichalcogenides, e.g., $\mathrm{MoS}_{2}{ }^{9,11-14}, \mathrm{WS}_{2}{ }^{15,16}$, and $\mathrm{WSe}_{2}{ }^{17,18}$. These materials show many unique optical and electrical properties, e.g., indirect-to-direct bandgap transitions and valley polarization ${ }^{19,20}$, and enrich the number of building blocks that may be used for next-generation electronic and optoelectronic devices.

Although most research has focused on 2D transition metal dichalcogenides, recently 2D layered metal monochalcogenides, e.g., GaS, GaSe, and InSe, have attracted increasing interest ${ }^{21-25}$. Recent studies on these 2D metal monochalcogenides indicate that they have significantly different electronic and optoelectronic properties from transition metal dichalcogenides ${ }^{21-25}$. Gallium selenide $(\mathrm{GaSe})$ is a typical layered metal monochalcogenide, which has a direct bandgap energy of $\sim 2 \mathrm{eV}^{26}$ and generally shows p-type behavior ${ }^{27,28}$. Bulk GaSe crystals can be constructed conceptually by vertically stacking single layers which are held together by van der Waals forces, with different stacking modes giving rise to different bulk crystal structures, i.e., $\beta$-GaSe, $\varepsilon$-GaSe, $\gamma$-GaSe, and $\delta$-GaSe ${ }^{29}$. A single layer of $\mathrm{GaSe}$ is composed of covalently bonded $\mathrm{Se}-\mathrm{Ga}-\mathrm{Ga}-\mathrm{Se}$ atoms, with a lattice constant of $0.374 \mathrm{~nm}$ and $\mathrm{D}_{3 h}$ symmetry ${ }^{29,30}$. Bulk GaSe shows many interesting electrical and optical properties, e.g., anisotropic Hall-mobility ${ }^{31}$, electron-hole liquid behavior ${ }^{32}$, and up-conversion luminescence $^{33}$, and has been widely used in the fields of optoelectronics, nonlinear optics, and terahertz radiation ${ }^{34-36}$. Recently, both theoretical and experimental work have revealed many new properties as bulk GaSe has been thinned systematically to $2 \mathrm{D}$ crystals. Theoretical studies have predicted that the bandgap of GaSe may be widely tuned by varying the numbers of layer in the crystal or by the inducing mechanical strain ${ }^{37-39}$. In addition, the low 

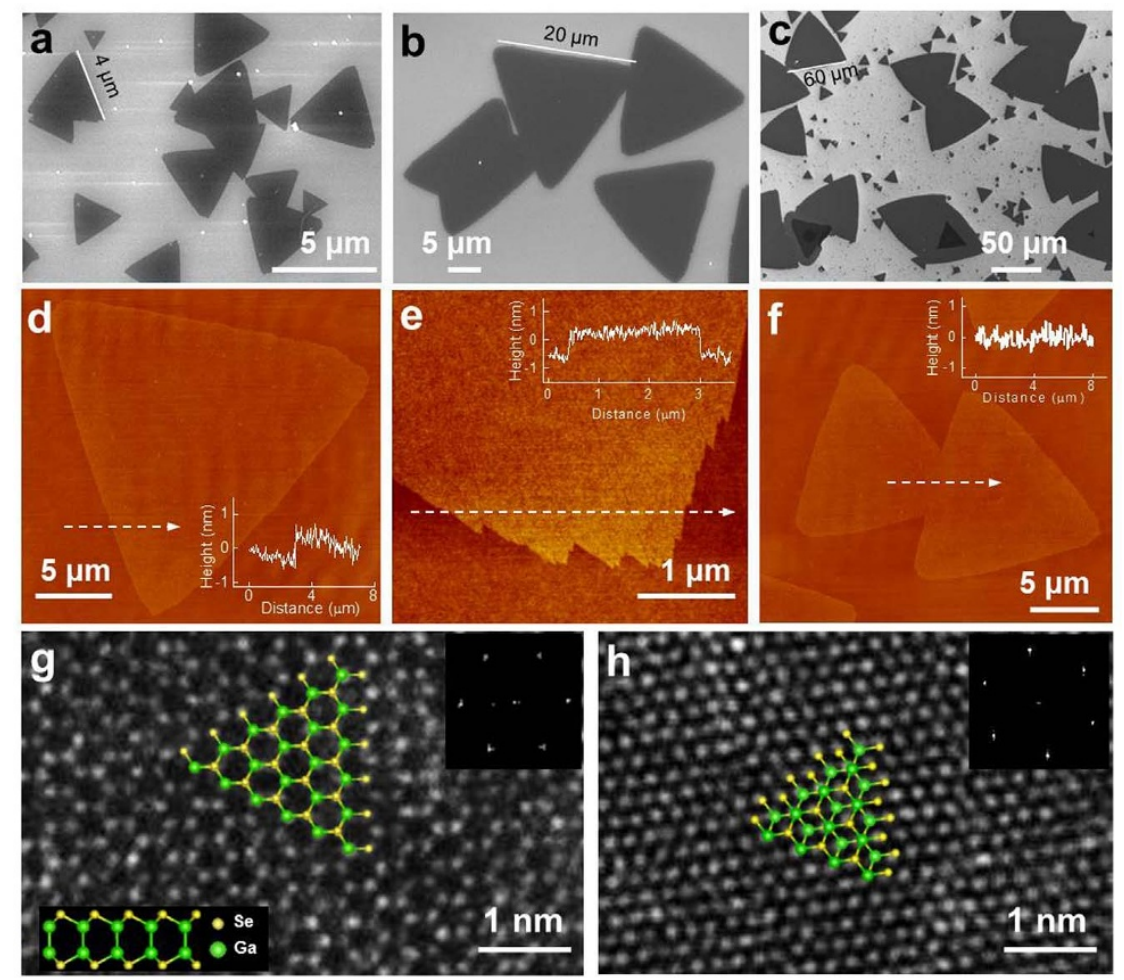

Figure 1 Growth of 2D GaSe crystals. (a-c) SEM images of monolayer triangular GaSe crystals grown for 2 min (a), 5 min (b), and 10 min (c). (d-f) AFM images of monolayer GaSe crystals. Insets are line profiles in the direction of the dashed arrows. Images (d) and (f) show an individual and two merged triangular monolayer flakes, respectively, while image (e) shows an enlarged view of the tip of the triangle. (g) High-resolution ADF-STEM image of monolayer GaSe. The lattice is composed of hexagonal rings of gallium and selenium atoms. Top and side views of monolayer GaSe structure are overlaid. Inset is the corresponding FFT image. (h) High-resolution ADF-STEM image of multi-layer GaSe. Top view of multi-layer GaSe structure with $\varepsilon$-type stacking is overlaid. Inset is the corresponding FFT image.

formation energy and band edge positions of monolayer GaSe makes it a suitable photocatalyst for solar water-splitting ${ }^{40}$. GaSe nanosheets with several atomic layers have been fabricated through mechanical exfoliation ${ }^{22,23}$, and show good optical and electrical performance as photodetectors and FETs, revealing the great potential of this $2 \mathrm{D}$ nanomaterial for such devices.

In order to realize practical uses of $2 \mathrm{D}$ materials-based devices, it is essential to synthesize large-area, high-quality $2 \mathrm{D}$ crystals with controlled layer numbers on various insulating and conducting substrates. To this end, 2D materials are generally fabricated by mechanical or chemical exfoliation from their layered bulk counterparts, or by vapor phase deposition, which includes thermal evaporation and chemical vapor deposition (CVD). Mechanically exfoliated 2D crystals generally have higher quality, but they are limited in size (usually up to tens of microns) and the thickness is not uniform and controllable. The vapor phase deposition method, on the other hand, has been widely used to grow large-area graphene and h-BN nanosheets ${ }^{10,41}$, as well as large, single crystal, monolayer semiconducting 2D materials such as $\mathrm{MoS}_{2}$ and $\mathrm{WS}_{2}{ }^{12-16}$. Although recently few-layer GaSe $2 \mathrm{D}$ crystals (up to $\sim 20 \mu \mathrm{m}$ in lateral size) have been synthesized on $\mathrm{SiO}_{2} / \mathrm{Si}$ substrates through vapor-phase mass transport, the use of nucleation seeds and the growth in a sealed quartz tube makes control of the uniformity and layer number of $2 \mathrm{D}$ GaSe crystals difficult ${ }^{24}$. To date, it is still a challenge to synthesize large and uniform 2D GaSe crystals with controllable layer numbers.

Here, we synthesized monolayer and multi-layer 2D GaSe crystals directly on $\mathrm{SiO}_{2} / \mathrm{Si}$ substrates using a vapor phase deposition method, in which large (up to $\sim 60 \mu \mathrm{m}$ in lateral size), uniform, single-crystalline monolayer GaSe crystals were obtained for the first time. The structure and orientation of 2D GaSe crystals are characterized from the atomic scale to micrometer scale by using scanning transmission electron microscopy (STEM) and dark-field transmission electron microscopy (DF-TEM). The well-defined 2D crystals were controllable by tuning growth conditions. The Raman spectra of the $2 \mathrm{D} \mathrm{GaSe}$ crystals change with their layer numbers in both peak positions and intensities. The Raman and theoretical modeling of the electronic structure of 2D GaSe crystals demonstrate a direct-toindirect bandgap transition and a progressive confinement-induced bandgap shift, which are significantly different from the widely studied transitional metal dichalcogenides such as $\mathrm{MoS}_{2}$. These 2D GaSe crystals show a high photoresponse with white light illumination, and great potential for next-generation electronic and optoelectronic devices.

\section{Results and Discussion}

The 2D GaSe crystals were synthesized in a tube furnace system equipped with a $1^{\prime \prime}$ diameter quartz tube (Figure S1). A mixture of bulk GaSe (Figure S2) and $\mathrm{Ga}_{2} \mathrm{Se}_{3}$ powders (molar ratio: 50:1) was thermally evaporated at $750^{\circ} \mathrm{C}$ under a pressure of 30 Torr and an argon carrier gas flow rate of 50-100 sccm (standard cubic centimeter per minute). The use of a small amount of $\mathrm{Ga}_{2} \mathrm{Se}_{3}$ provides sufficient Se for growing regularly-shaped triangle monolayer flakes, which will be discussed in detail below. Compared with previous approaches to synthesize 2D GaSe through vapor-phase mass transport within sealed tubes ${ }^{24}$, the precise control over the gas flow rate and reaction chamber pressure in this study allowed reproducible and controllable correlation between growth conditions and resulting nanostructure. The $2 \mathrm{D}$ products were deposited on $\mathrm{SiO}_{2}$ $(\sim 300 \mathrm{~nm}) / \mathrm{Si}$ substrates (See Methods for detailed synthesis process). Figure 1a-c show typical scanning electron microscopy (SEM) images of the $2 \mathrm{D}$ products synthesized at the growth temperature from $\sim 710$ to $\sim 720^{\circ} \mathrm{C}$, with an argon gas flow rate of $50 \mathrm{sccm}$, and 
growth times of $2 \mathrm{~min}$ (Figure 1a), $5 \mathrm{~min}$ (Figure 1b), and $10 \mathrm{~min}$ (Figure 1c). The products are triangular flakes, some of which merge together to form irregularly-shaped islands. The triangular flakes are composed of $\mathrm{Ga}$ and Se with an atomic ratio of $1: 1(\mathrm{GaSe})$ as determined by energy-dispersive $x$-ray spectroscopy (EDS) (Figure S3; the bright, small particles on the flakes are Se nanoparticles deposited during the synthesis, and can be removed by heat treatment at $300^{\circ} \mathrm{C}$ in vacuum). The lateral size of the triangular flakes increases with increasing growth time, from up to $\sim 4 \mu \mathrm{m}$ for $2 \mathrm{~min}$ (Figure 1a), to $\sim 20 \mu \mathrm{m}$ for $5 \mathrm{~min}$ (Figure $1 \mathrm{~b}$ ), and to $\sim 60 \mu \mathrm{m}$ for $10 \mathrm{~min}$ (Figure 1c). The average lateral size of the triangular flakes depending on growth times are presented in Figure S4. Except for only a few thicker patches with darker contrast, the largely uniform contrast in the SEM images reveal that most of the GaSe domains have uniform thickness (Figure 1c).

The detailed morphology and thickness of the GaSe flakes were characterized by atomic force microscopy (AFM). Figure 1d shows the AFM image of an individual triangular flake, which is equilateral with sharp and smooth edges. A dashed-line arrow across the edge shows that the thickness of the flake is $\sim 0.8 \mathrm{~nm}$, corresponding to a single atomic layer of $\mathrm{GaSe}^{38}$. Statistics from a series of SEM images covering the whole substrate indicates that more than $90 \%$ of the GaSe domain is monolayer. The result demonstrates that large, uniform monolayer GaSe crystals were grown on the $\mathrm{SiO}_{2} / \mathrm{Si}$ substrate for the first time (the darker domains shown in Figure 1c correspond to multi-layer flakes, which will be discussed in detail later (Figure 3)). An enlarged AFM image indicates that the area around the tip of the triangle has more irregularities when compared with the side of the triangle (Figure 1e). Such jagged edges were also observed in other $2 \mathrm{D}$ monolayer materials ${ }^{12,16}$. Figure $1 \mathrm{f}$ shows the AFM image of two merged monolayer GaSe flakes. This domain is uniform in thickness, indicating that when the two monolayer GaSe flakes made contact, they merged instead of one overgrowing the other.

The crystal structures of our vapor-phase grown 2D GaSe were characterized using aberration-corrected STEM and electron diffraction. The 2D samples for structural characterizations were grown directly on amorphous silicon films (5 $\mathrm{nm}$ in thickness) supported by a silicon TEM grid under the same conditions as described above. The atomic structure of the monolayer GaSe flake is shown with annular aberration-corrected dark-field STEM (ADF-STEM) imaging in Figure 1g. Hexagonal rings composed of Ga and Se atoms are clearly visible, in agreement with the hexagonal structure of monolayer GaSe (as indicated by the top- and side-view schematics in Figure $1 \mathrm{~g}$ ). The distance between two in-plane adjacent $\mathrm{Ga}$ (or Se) atoms is $\sim 0.38 \mathrm{~nm}$, matching the lattice parameters of the $a-b$ plane $(0.375 \mathrm{~nm})$ in $\mathrm{GaSe}$ crystals $^{29}$, but it is hard to distinguish $\mathrm{Ga}$ and Se atoms in the ADF-STEM image due to their similar atomic numbers $^{13}$, and because the GaSe flakes were imaged on top of amorphous silicon membranes, which results in a reduction of contrast. The corresponding Fast Fourier Transforms (FFT) of the image (inset of Figure 1g) confirms the hexagonal structure of the monolayer GaSe. In contrast to the hexagonal-ring structure of monolayer GaSe crystals, multi-layer GaSe flakes generally show a close-packed structure along $c$-axis as shown in Figure $1 \mathrm{~h}$. Such a structure indicates that the stacking of the multi-layer crystal can be $\varepsilon^{-}, \gamma_{-}$, or $\delta$ type, but not $\beta$-type, as indicated by top-view schematic in Figure $1 \mathrm{~h}$.

The crystallinity, grain orientation, and grain boundary of $2 \mathrm{D}$ monolayer GaSe flakes were studied by selected-area electron diffraction (SAED) and DF-TEM. Figure 2a shows the bright-field TEM (BF-TEM) image of a single monolayer triangular flake. Note that the triangles grown on the $5 \mathrm{~nm}$-thick amorphous silicon film are not as sharp and smooth as those grown on $\mathrm{SiO}_{2} / \mathrm{Si}$ substrates (Figure 1), indicating that the substrate may play an important role in determining the shape of the monolayer flakes. The SAED pattern obtained from the flake in Figure 2a shows only one set of six-fold symmetry diffraction spots (inset of Figure $2 a$ ), indicating that the
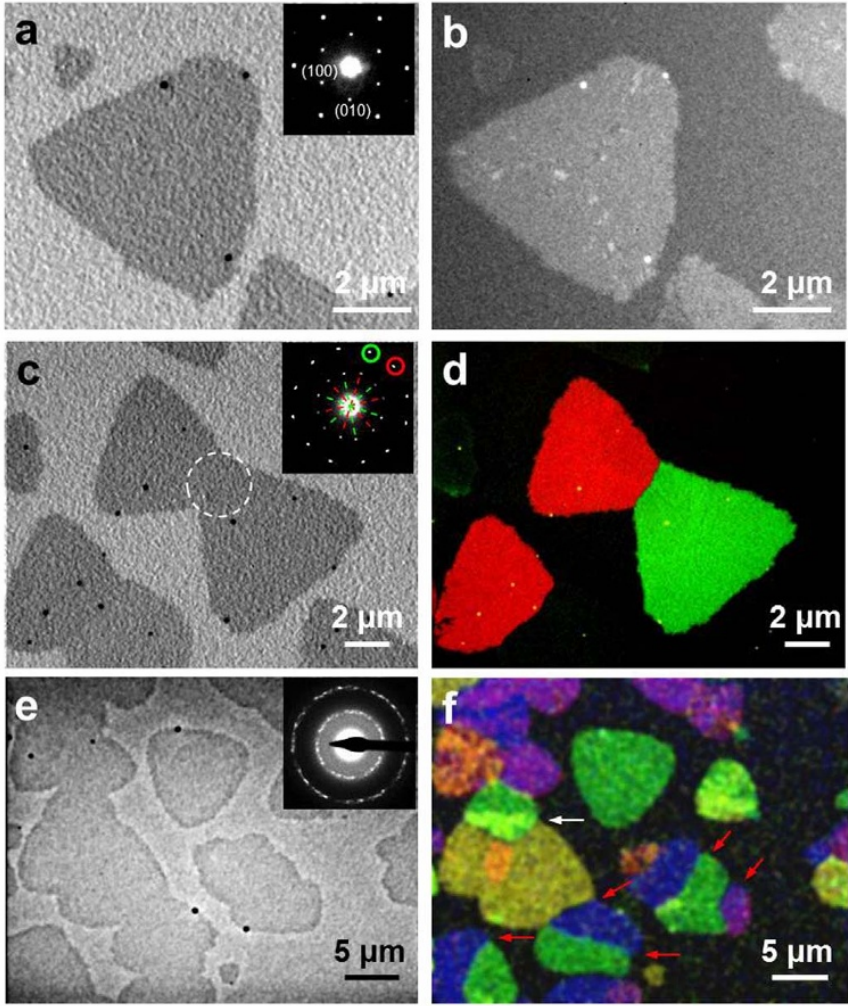

Figure $2 \mid$ Grain structures in monolayer GaSe crystals. (a) Bright-field TEM image of a single monolayer triangular flake. Inset is the SAED pattern of the flake, showing a single set of spots in a hexagonal pattern. (b) DF-TEM image of the flake in (a). (c) Bright field TEM image showing two monolayer triangular flakes merging together. Inset is the SAED pattern obtained from the common area of the two flakes as indicated by a dashed circle. The pattern shows two sets of spots in a hexagonal pattern (indicated by red and green dashed-lines, respectively) with orientated $\sim 30^{\circ}$ apart. (d) Color-coded overlay of DF-TEM images corresponding to the red- and green-circled diffraction spots in the inset of (c). (e) Brightfield TEM image of an area containing both monolayer triangular flakes and large islands of merged flakes. Inset is the electron diffraction pattern obtained from the whole area in (e). (f) Color-coded overlay of DF-TEM image of the area in (e). The overlapped crystal grains are indicated by the white arrows and the clear grain boundaries are indicated by the red arrows.

flake is single crystal with a hexagonal crystal structure. The corresponding DF-TEM image further confirms the single-crystal nature of the triangle GaSe flake (Figure 2b). The BF-TEM image in Figure $2 \mathrm{c}$ highlights a pair of merged monolayer triangle flakes with a crystal misorientation of $\sim 30^{\circ}$ as indicated by two sets of hexagonal diffraction patterns in the inset of Figure $2 c$. The angle-resolved, false color DF-TEM image (Figure 2d), made by overlaying two colorcoded DF-TEM images acquired from the red- and green-circled diffraction spots, respectively, demonstrates that the intersection of the two grains forms a very sharp boundary. Figure 2e shows the BFTEM image of a region containing both isolated triangle flakes and islands of merged flakes. The electron diffraction pattern (inset of Figure 2e) acquired from all the flakes in Figure 2e shows typical polycrystalline rings, indicating the flakes have different crystallographic orientations. This can be visualized in the false color DFTEM image in Figure $2 \mathrm{f}$, wherein different crystallographic orientations shown in the electron diffraction pattern in Figure 2e correspond to different colors. The result indicates that the irregularlyshaped islands are comprised of single crystal flakes having different orientations, with most of them connected to each other by grain boundaries (indicated by red arrows in Figure $2 \mathrm{f}$ ), and only a few 


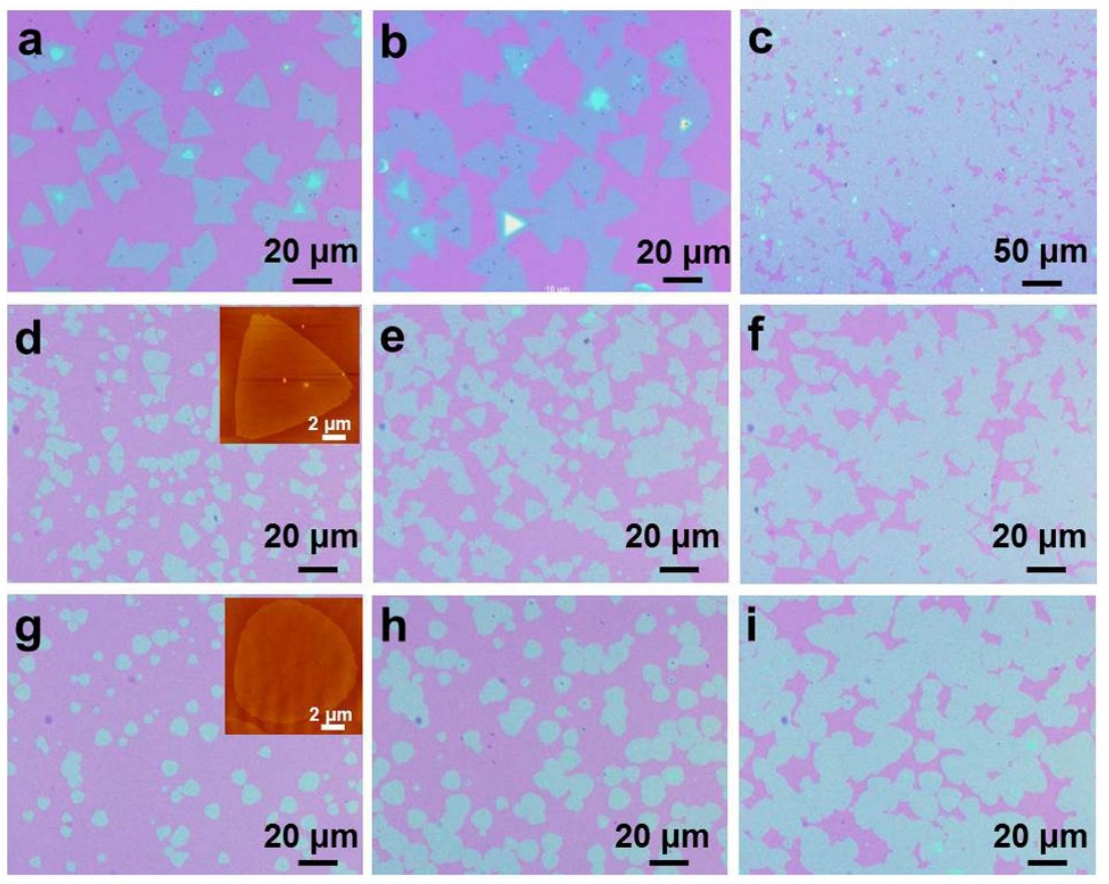

Figure 3 Influence of growth conditions on monolayer GaSe crystals. Optical micrographs of 2D monolayer GaSe crystals synthesized at a growth temperature of $(\mathrm{a}-\mathrm{c}) \sim 710-720^{\circ} \mathrm{C},(\mathrm{d}-\mathrm{f}) \sim 700-710^{\circ} \mathrm{C}$, and $(\mathrm{g}-\mathrm{i}) \sim 660-670^{\circ} \mathrm{C}$, with an argon gas flow rate of 50 sccm and a growth time of 5 min. The images were obtained near the downstream side ( $a, d, g)$, middle (b, e, h), and upstream side (, , f, i) of the substrates. Insets of (d) and (g) are AFM images of individual crystals.

overlapping to form bilayer regions (indicated by a white arrow in Figure 2f).

In our vapor-phase growth process, the size, density, shape, thickness, and uniformity of the resulting 2D GaSe crystals were strongly influenced by the growth region in the furnace, growth temperature, and argon carrier gas flow rate. Figures $3 \mathrm{a}-\mathrm{c}$ show optical micrographs of the monolayer GaSe synthesized at growth temperatures from $\sim 710$ to $\sim 720^{\circ} \mathrm{C}$, with an argon gas flow rate of $50 \mathrm{sccm}$, and a growth time of $5 \mathrm{~min}$. The three images were obtained from three different regions on the $\mathrm{SiO}_{2} / \mathrm{Si}$ substrate, i.e., close to the downstream side (right side of the substrate as shown in Figure S1) (Figure 3a), around the middle (Figure 3b), and close to the upstream side (left side of the substrate as shown in Figure S1) (Figure 3c). The images clearly show that for growth regions closer to the upstream side of the furnace, the sizes of the triangular flakes increased and more islands of merged triangles were formed, with all the flakes merging into a continuous film in the region closest to the upstream side of the substrate. Such changes in size and density of the monolayer GaSe crystals with different growth regions may be caused by the temperature gradient and the change in diffusion flux of the source along the substrate ${ }^{42}$. In all cases studied, if the substrate was placed at a lower temperature region, i.e., $\sim 700-710^{\circ} \mathrm{C}$ (Figure $3 \mathrm{~d}-\mathrm{f}$ ) and $\sim 660-670^{\circ} \mathrm{C}$ (Figure $3 \mathrm{~g}-\mathrm{i}$ ), the synthesized monolayer GaSe crystals followed the same trend, merging into a continuous layer in the upstream region of the substrate due to higher nucleation density. Note that the gas flow in our growth tube is laminar - i.e., Reynold's number $\sim 2.2$ (see Supporting Information for detailed calculation $)^{42}$ - and this, taken with the results above, indicate the growth of monolayer GaSe was controlled mainly by a diffusion process through a boundary layer, the thickness of which plays an important role in controlling the diffusion flux ${ }^{42}$. In the present case, the diffusion flux on the substrate is inversely proportional to the distance from the side of the substrate closest to the source materials ${ }^{42}$, and a since higher diffusion flux generates more nucleation sites and favors higher growth rate, the GaSe flakes grown near the upstream side of the substrate (closer to the source) show larger size and higher density than those near the downstream side.

It has already been shown by structural characterization that the shape of monolayer GaSe flakes may be influenced by the growth substrate. In fact, the shape of monolayer GaSe is also sensitive to growth temperature. As already demonstrated by the results in Figure 1 and Figures $3 a-c$, equilateral triangular monolayer flakes with smooth and straight edges were grown at $\sim 710-720^{\circ} \mathrm{C}$. However, when the growth temperature was reduced (with all other growth conditions unchanged) to $\sim 700-710^{\circ} \mathrm{C}$, equilateral triangular monolayer GaSe flakes were still obtained (Figure 3d-f), but with edges that are not as linear as those grown at $\sim 710-720^{\circ} \mathrm{C}$. Further decreases in the growth temperature, i.e., at $\sim 660-670^{\circ} \mathrm{C}$, resulted in monolayer GaSe flakes that were almost round (Figure $3 \mathrm{~g}-\mathrm{i}$ ), and the change of the shape at different growth temperatures might be explained by the minimum energy shape of monolayer $\mathrm{GaSe}^{43}$. Based on the 3-fold symmetry of a single atomic layer of $\mathrm{GaSe}^{29,30}$, the minimum energy shape is expected to be a triangle ${ }^{43}$. However, at low growth temperatures depositing GaSe molecules may be captured immediately when encountering a growing nucleus, and lack sufficient energy to desorb and move to energetically preferred location. As deposition and surface diffusion are random, rounded flakes are necessarily formed. In contrast, at higher growth temperature the depositing GaSe molecules have enough mobility to desorb and move from random, high energy positions to minimum energy positions, thereby favoring triangular flakes. As shown in Figure S5, rounded monolayer GaSe flakes were also formed when only GaSe was used as the source material (without the small amount of $\mathrm{Ga}_{2} \mathrm{Se}_{3}$ powders), and may be explained by considering the concentration of vapor-phase reactant species in the growth region. During the thermal evaporation process, bulk GaSe decomposes into vapor-phase $\mathrm{Ga}_{2} \mathrm{Se}$ and $\mathrm{Se}_{2}$, which then diffuse to the substrate with different velocities and mean free paths ${ }^{44}$ (this could be the reason for the deposition of Se nanoparticles on 2D GaSe flakes as observed in SEM images). The lack of one species may impede the growth of 

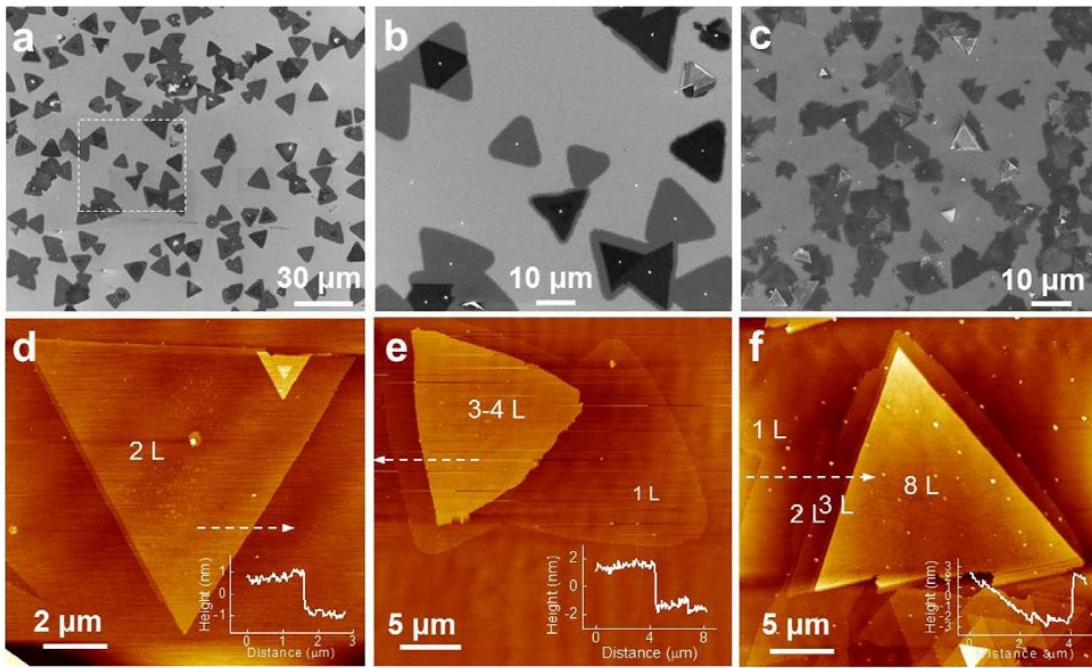

Figure $4 \mid$ Multi-layer 2D GaSe crystals. (a-c) SEM images of GaSe crystals grown with an 80 sccm argon flow for 5 min. Images (a) and (b) were obtained from the region close to the downstream side of the substrate. The flakes with lighter contrast are monolayer, while darker flakes indicate additional layers grown on monolayer flakes. (b) is the enlarged image of the area contained in the dashed square in (a). Image (c) shows thicker multilayer GaSe crystals on a continuous monolayer GaSe film grown in the region close to the upstream side of the substrate. (d-f) AFM images of multi-layer GaSe crystals. Insets are line profiles along the dashed arrows.

the $2 \mathrm{D}$ crystals as seen in previous reports wherein GaSe and $\mathrm{MoS}_{2}$ flakes grown by vapor-phase deposition were transformed from regular triangles to truncated triangles and even to hexagons as the concentration of selenium or sulfur was reduced ${ }^{14,24}$. Therefore, it is possible in the present case that the concentration of $\mathrm{Se}_{2}$ is lower than $\mathrm{Ga}_{2} \mathrm{Se}$ in the growth region when only GaSe is used, and the addition of $\mathrm{Ga}_{2} \mathrm{Se}_{3}$ increases the $\mathrm{Se}_{2}$ concentration, resulting in regularlyshaped triangle flakes. It should be noted that the amount of $\mathrm{Ga}_{2} \mathrm{Se}_{3}$ must be well controlled, with the best molar ratio of $\mathrm{GaSe} /$ $\mathrm{Ga}_{2} \mathrm{Se}_{3}$ being $\sim 50 / 1$ (larger amounts of $\mathrm{Ga}_{2} \mathrm{Se}_{3}$ resulted in the formation of $\mathrm{Ga}_{2} \mathrm{Se}_{3}$ crystals on the substrate).

The argon gas flow rate played an important role in controlling the number of layers in the 2D GaSe flakes. The results in Figure 1 and Figures $3 \mathrm{a}-\mathrm{c}$ have already shown that uniform monolayer GaSe flakes ( $>90 \%$ of all the GaSe domains) were grown with an argon gas flow rate of $50 \mathrm{sccm}$. However, the quantity of multi-layer flakes can be increased deliberately by increasing the argon gas flow rate. Figures $4 \mathrm{a}-\mathrm{c}$ show SEM images of $2 \mathrm{D}$ GaSe crystals grown with $80 \mathrm{sccm}$ argon gas flow and a growth time of $5 \mathrm{~min}$. Isolated and merged monolayer (in lighter contrast) and multi-layer (in darker contrast) flakes are observed near the downstream side of the substrate (Figure $4 \mathrm{a}, \mathrm{b}$ ). The monolayer domains in this region decrease to $\sim 60 \%$ of all the GaSe domains, and the multi-layer flakes generally have 2-4 layers (2-4 L) as determined by AFM (Figure $4 \mathrm{~d}$, e). As shown in the SEM images (Figure $4 \mathrm{a}, \mathrm{b}$ ), the size of the multi-layer triangular flakes are generally smaller than the underlying monolayer ones (multi-layer flakes truncated as they grew to the edges of monolayer flakes can be observed, as shown in Figure $4 \mathrm{~b}$ ). With the growth region moving towards the upstream side of the substrate, a higher density of thicker (up to $\sim 8-15 \mathrm{~L}$ ) multi-layer GaSe flakes were grown on what appears to be a continuous film of monolayer $\mathrm{GaSe}$ (Figure 4c, f). These results indicate that the growth of multi-layer GaSe crystals is likely governed by a 'layer-plus-island' growth mode ${ }^{45}$, and a higher argon carrier gas flow rate, which results in a higher overall diffusion flux of the source ${ }^{42}$, favors the growth of multi-layer flakes. Indeed, further increasing the argon gas flow rate, i.e., to $100 \mathrm{sccm}$, led to more and much thicker (up to $\sim 55 \mathrm{~L}$ ) multilayer GaSe flakes on continuous monolayer films (Figure S6). However, when the argon gas flow rate was lowered, i.e., to $20 \mathrm{sccm}$, no in-plane (horizontal to the substrate) monolayer and multi-layer GaSe flakes were obtained. Instead, multi-layer GaSe flakes grew vertically on the substrate, forming 'flower-like' structures (Figure S7).

One of the most prominent characteristics of 2D materials is their physical properties depending strongly on layer number. In this work, we studied the optical properties of our 2D GaSe crystals with different layer numbers using Raman spectroscopy. Figure 5a shows the AFM image of 2D multi-layer GaSe crystals with different layer numbers grown on a continuous monolayer film (see also Figure S8 with line profiles determining layer numbers). The Raman spectrum (measured with $532 \mathrm{~nm}$ laser excitation) of a GaSe crystal with $\sim 30$ L shows peaks at $\sim 136.7 \mathrm{~cm}^{-1}, 215.6 \mathrm{~cm}^{-1}, 244.1 \mathrm{~cm}^{-1}$, and $309.3 \mathrm{~cm}^{-1}$, corresponding to the $\mathrm{A}_{1 \mathrm{~g}}^{1}, \mathrm{E}^{1}{ }_{2 \mathrm{~g}}, \mathrm{E}_{1 \mathrm{~g}}^{2}$, and $\mathrm{A}_{1 \mathrm{~g}}^{2}$ vibration mode of $\mathrm{GaSe}$, respectively (Figure $5 \mathrm{~b}$, green curve ${ }^{46}$. As shown in Figure $5 b$, the intensity of these Raman peaks decreases with the reduction of layer numbers as shown by the Raman spectra of GaSe crystals with 12 L (pink curve), 7 L (blue curve), 3 L (red curve) and $1 \mathrm{~L}$ (purple curve). Note that the Raman spectrum of monolayer GaSe $(1 \mathrm{~L})$ is almost the same as that of $\mathrm{Si}$ from the $\mathrm{SiO}_{2} / \mathrm{Si}$ substrate (Figure 5b, black curve). This phenomenon was also observed in exfoliated $\mathrm{GaSe}$ nanosheets $\mathrm{s}^{22,47}$. In addition to the intensity, the Raman peaks also show shifts with the change in layer numbers. The $\mathrm{A}^{1}{ }_{1 \mathrm{~g}}$ peak, corresponding to the out-of-plane mode, is located at $\sim 136.7 \mathrm{~cm}^{-1}$ for the GaSe crystal with $\sim 30 \mathrm{~L}$, at $\sim 135.3 \mathrm{~cm}^{-1}$ for $12 \mathrm{~L}, \sim 133.8 \mathrm{~cm}^{-1}$ for $7 \mathrm{~L}$, and $\sim 131.1 \mathrm{~cm}^{-1}$ for $3 \mathrm{~L}$ (hardly observed for $1 \mathrm{~L}$ ). The red-shift of the peak with the decrease in layer numbers is probably due to the decrease in inter-layer interaction. Another peak associated with the out-of-plane mode, the $\mathrm{A}^{2}{ }_{1 \mathrm{~g}}$ peak, also shows red-shift from $\sim 309.3 \mathrm{~cm}^{-1}$ for the $\sim 30 \mathrm{~L}$-crystal to $\sim 306.5 \mathrm{~cm}^{-1}$ for the $7 \mathrm{~L}$-crystal; however, it is hard to study the shift of $\mathrm{A}^{2}{ }_{1 \mathrm{~g}}$ peak for thinner GaSe flakes (e.g., $1 \mathrm{~L}$ and $3 \mathrm{~L}$ ) because of the increasing Raman signal from the substrate, e.g., the peak at $\sim 303.3 \mathrm{~cm}^{-1}$ from $\mathrm{Si}$, which overlaps with the spectra of GaSe. A Raman map of the $7 \mathrm{~L}$ - and $12 \mathrm{~L}-\mathrm{GaSe}$ crystals (included in the dashed square in Figure 4a) was obtained by monitoring the $\mathrm{A}^{1}{ }_{1 \mathrm{~g}}$ peak (Figure 5c), and except for some brighter spots on the edge of the flakes, which originate from small, thicker crystals as shown in Figure $5 \mathrm{a}$, the intensities were uniform within the terrace region, suggesting good quality crystals.

To understand the effect of dimensional confinement on the electronic properties of $2 \mathrm{D}$ GaSe crystals, theoretical calculation of the electronic band structures was performed. The electronic band struc- 

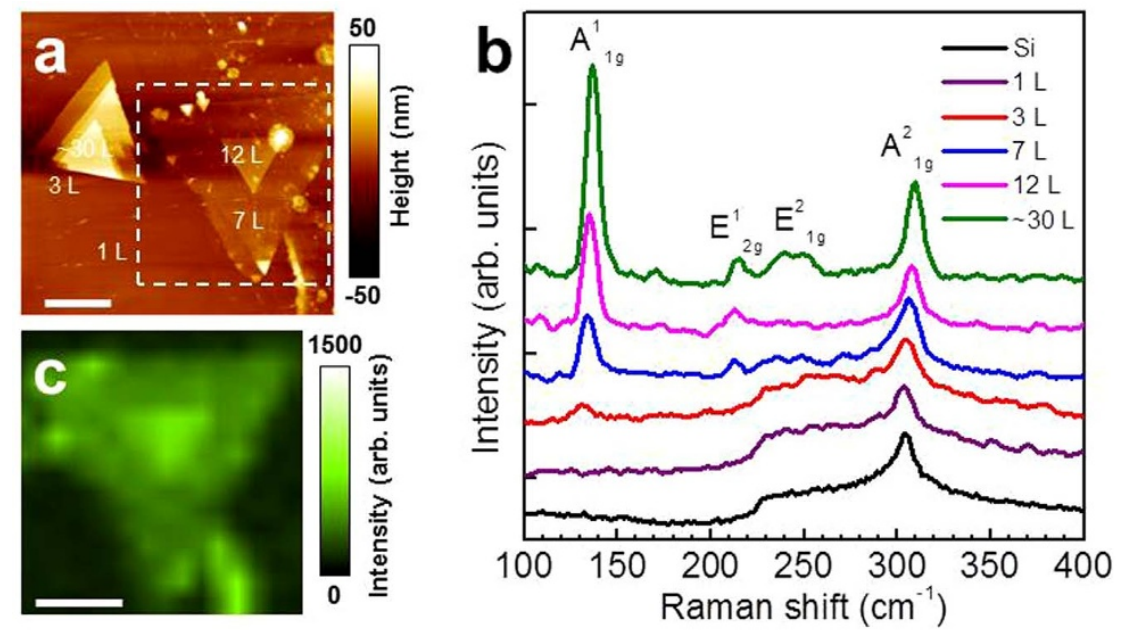

Figure 5 Optical properties of 2D GaSe. (a) AFM image of 2D GaSe crystals with different layer numbers (from 1 to $\sim 30 \mathrm{~L}$ ). The scale bar is $5 \mu$ m. (b) Raman spectra (532 nm laser excitation) of 2D GaSe crystals with 1, 3, 7, 12, and $\sim 30 \mathrm{~L}$ as indicated in (a) and a bare substrate. Note that the spectra were offset for clarity. (c) Raman mapping of the crystals included in the dashed square in (a) by monitoring $\mathrm{A}^{1}{ }_{1 \mathrm{~g}}$ peak in the Raman spectra. The scale bar is $5 \mu \mathrm{m}$.

tures of 2D GaSe crystals were investigated using a highly accurate, all-electron first-principles quantum mechanical calculation code $\left(\right.$ FHI-aims $\left.{ }^{48}\right)$. The exchange-correlation potential of the PerdewBurke-Ernzerhof (PBE) version of the generalized-gradient approximation (GGA) ${ }^{49}$ was used. Figure 6 a shows the electronic band structures of monolayer GaSe and its $\varepsilon$-type bulk counterpart, with respect to their valence band maximum (VBM) (see Supporting Information for detailed calculation process). Both the VBM and the conduction band minimum $(\mathrm{CBM})$ are located at the $\Gamma$ point for bulk, which means that bulk $\varepsilon$-GaSe has a direct bandgap, which agrees with previous calculations and experimental result ${ }^{26,40,50}$. However, with decreasing layer number $(<7 \mathrm{~L})$, as shown in Figure $6 \mathrm{~b}$, the VBM splits in a symmetric way along the $\Gamma$ point, and a progressive confinement-induced bandgap shift for $2 \mathrm{D}$ crystals occurs, indicating that these crystals have an indirect bandgap and its energy increases with decreasing layer numbers (Figure 6c). This is consistent with the blue-shifted emission bands of $2 \mathrm{D}$ GaSe crystals observed in exfoliated 2D GaSe nanosheets ${ }^{23}$. The theory-predicted direct-to-indirect bandgap transition with decreasing layer number of 2D GaSe also contribute to the decreasing Raman intensity due to the suppressed inter-layer electron orbital coupling ${ }^{51}$. These findings demonstrate that 2D GaSe crystals are significantly different with the widely studied $\mathrm{MoS}_{2}$, which has an indirect-to-direct bandgap transition in monolayer ${ }^{11-14,19}$. Moreover, the energy difference between the direct gap and indirect gap for monolayer GaSe is so small that electrons can easily move between the minima with a small amount of thermal energy.
The large scale vapor phase growth of 2D GaSe crystals directly on device-compatible $\mathrm{SiO}_{2}$ substrates is favorable for conventional lithographic processes since this method does not need an extra transfer step, a step that may cause damage and contamination to the mechanically delicate $2 \mathrm{D}$ crystals. We used a standard e-beam photolithography process to make devices on 2D GaSe crystals grown on $\mathrm{SiO}_{2} /\left(\mathrm{p}^{++}\right) \mathrm{Si}$ substrates as illustrated in Figure 7a. Unfortunately, it is hard to get electrical signals from devices fabricated on monolayer GaSe. Figure $7 \mathrm{~b}$ shows the optical micrograph of a few-layer GaSe-based device with patterned electrodes (see also Figure S9a). Electrical characterization was carried out both in darkness and under the illumination (white light, $1.2 \mathrm{~mW} / \mathrm{cm}^{2}$ ). The current was measured using two electrodes as indicated by the red arrows in Figure $7 \mathrm{~b}$, and the effective area of this 2D GaSe-based device was $\sim 5.73 \mu \mathrm{m}^{2}$. Figure $7 \mathrm{c}$ shows the drain-source $\left(\mathrm{I}_{\mathrm{ds}}-\mathrm{V}_{\mathrm{ds}}\right)$ characteristic of the device in the dark (black curve) and under white light illumination (red curve), and the $\mathrm{I}_{\mathrm{ds}}-\mathrm{V}_{\mathrm{ds}}$ curves are linear (Inset of Figure 7c) and symmetric for small bias voltages (see Figure S9b), indicating Ohmic contacts. At a $\mathrm{V}_{\mathrm{ds}}$ of $-10 \mathrm{~V}$, the $\mathrm{I}_{\mathrm{ds}}$ of the device increased by three orders of magnitude (relative to the dark current) when exposed to white light illumination, indicating good photoresponse. Note that the photocurrent $\left(\mathrm{I}_{\mathrm{ph}}\right)$ also increased with bias voltage $\mathrm{V}_{\mathrm{ds}}$ due to the increase in carrier drift velocity and related reduction of the carrier transit time. We also recorded the dependence of $\mathrm{I}_{\mathrm{ds}}$ on the back-gate voltage $\left(\mathrm{V}_{\mathrm{g}}\right)$ at a fixed $\mathrm{V}_{\mathrm{ds}}$ of $-10 \mathrm{~V}$ (Figure $7 \mathrm{~d}$ ). In the dark state, our device showed the typical behavior of a field-effect transistors with a p-type channel (black curve in
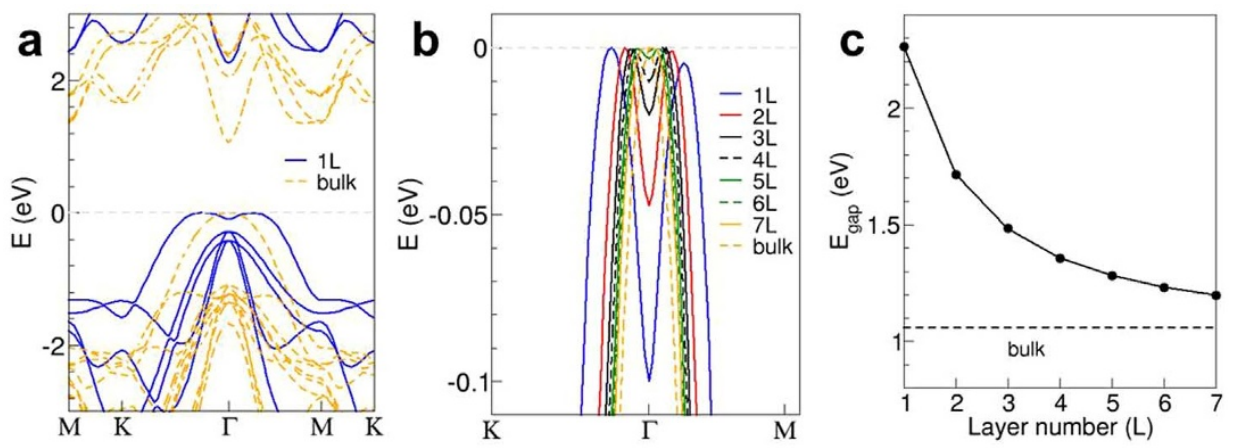

Figure 6 | Electronic band structures of 2D GaSe from theoretical calculations. (a) Energy band plots of monolayer and bulk GaSe along the high symmetry k-points. (b) Energy bands near the valence band maximum. (c) Bandgap energy as a function of layer numbers. 

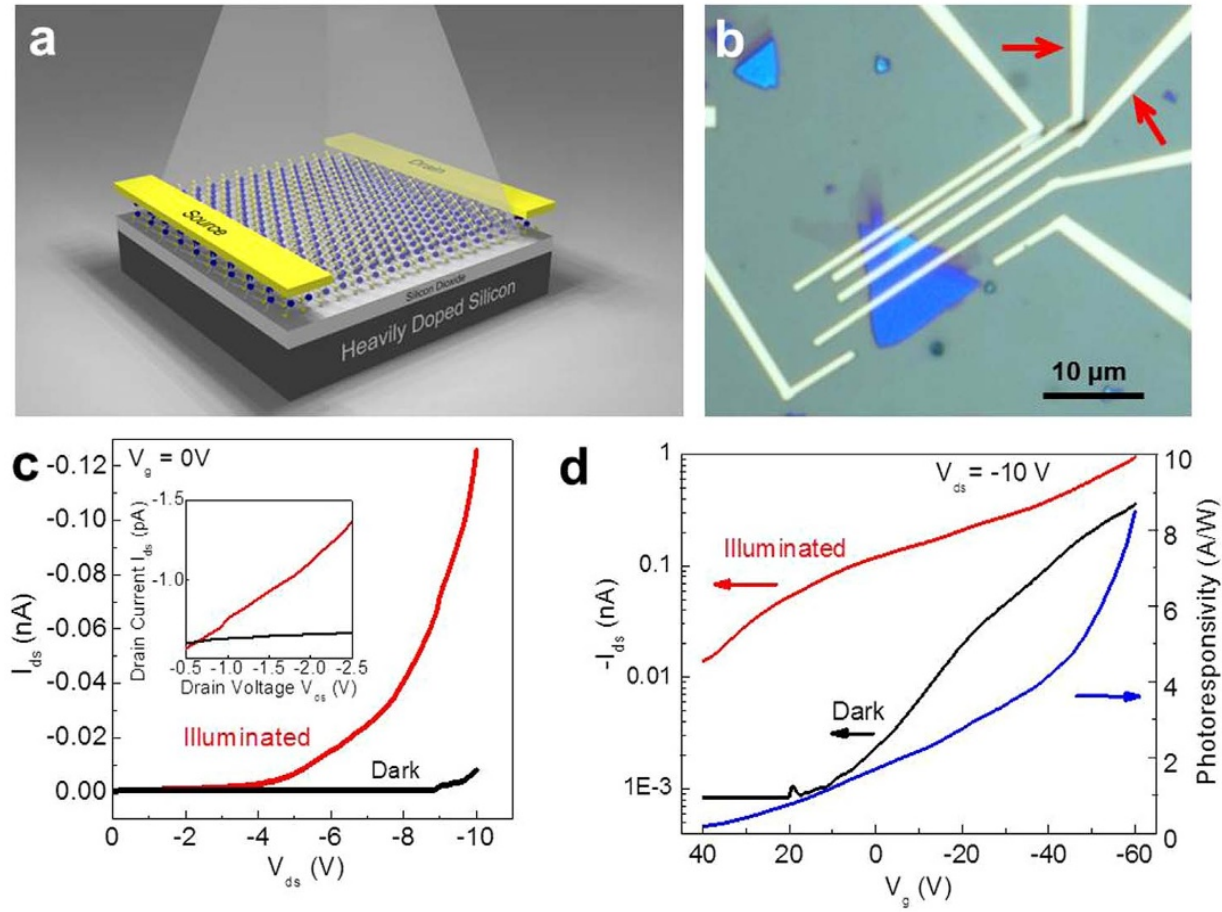

Figure $7 \mid$ Photoconducting properties of 2D GaSe crystals. (a) Illustration of a 2D GaSe-based device illuminated with white light. (b) Optical image of a 2D GaSe device with patterned electrodes. The red arrows indicate the two electrodes used for measurements. (c) $I_{d s}-V_{d s}$ characteristics of the 2D GaSe device in the dark (black curve) and with white light illumination (power density: $1.2 \mathrm{~mW} / \mathrm{cm}^{2}$ ) (red curve). (d) The transfer characteristics of the $2 \mathrm{D} \mathrm{GaSe}$ FET with $\mathrm{V}_{\mathrm{ds}}=-10 \mathrm{~V}$ in the dark (black curve) and with white light illumination (red curve). Calculated responsivity as function of the $\mathrm{V}_{\mathrm{g}}$ also shown (blue curve).

Figure 7d), but when illuminated by the white light source, the OFF current increased from $\sim 0.8 \mathrm{pA}$ to $\sim 14 \mathrm{pA}$, with the $\mathrm{I}_{\mathrm{ds}}$ increasing at both OFF and ON states for all values of $V_{g}$ (red curve in Figure $7 \mathrm{~d}$ ). This result indicates that the photocurrent dominated the thermonic and tunneling currents over the entire operating range of the device. According to the photocurrent generated under different $\mathrm{V}_{\mathrm{g}}$, the responsivity $\left(\mathrm{R}=\mathrm{I}_{\mathrm{ph}} / \mathrm{P}_{\text {light }}\right)$ was calculated and plotted as a function of $V_{g}$ (blue curve in Figure $7 d$ ). Under a fixed $V_{d s}$ of $-10 \mathrm{~V}$, the responsivity of the $2 \mathrm{D}$ GaSe crystal was $\sim 1.7 \mathrm{~A} / \mathrm{W}$ at zero gate voltage, and it increased to $8.5 \mathrm{~W} / \mathrm{A}$ at a gate voltage of approximately $-60 \mathrm{~V}$, indicating that the back gate plays an important role in tailoring the photocurrent in these crystals. The responsivity from our vapor phase grown GaSe $2 \mathrm{D}$ crystals is comparable to that of exfoliated few-layered GaSe nanosheets $(2.8 \mathrm{~A} / \mathrm{W})^{23}$ and higher than previously reported vapor-phase grown $2 \mathrm{D}$ GaSe $(17 \mathrm{~mW} / \mathrm{A})^{24}$. The low or lack of electrical response for monolayer of GaSe is consistent with the declined in Raman intensities for monolayer GaSe. The results suggest that monolayer GaSe might not be ideal for some optoelectronic properties due to tradeoffs between dimensional confinement and a transition from a direct to indirect bandgap. The detailed correlations between layer number and their optoelectronic properties are being studied.

In summary, large, single crystalline, uniform monolayer and multi-layer GaSe crystals were grown on $\mathrm{SiO}_{2} / \mathrm{Si}$ substrate through a vapor-phase growth method. The size, density, shape, thickness, and uniformity of the crystals were shown to be controllable during the synthesis process. The multiscale structural characterizations show the synthesized 2D GaSe crystals have highly crystallinity, distinct crystal orientation, and clear grain boundary. The theoretical modeling of the electronic structure and Raman spectroscopy demonstrate a direct-to-indirect bandgap transition and progressive confinement-induced bandgap shifts for 2D GaSe crystals. The 2D GaSe crystals show a high photoresponse and FET characteristics, which demonstrate the potential of this material for electronic and optoelectronic applications such as FETs and photodetectors. The successful synthesis of large, uniform monolayer and multi-layer GaSe crystals on $\mathrm{SiO}_{2} / \mathrm{Si}$ substrate demonstrates that our method can be used potentially for the growth of other semiconducting metal monochalcogenides, e.g., GaS, GaTe, InS, InSe, and InTe, further enriching the building blocks for the fabrication of $2 \mathrm{D}$ electronic and optoelectronic devices.

\section{Methods}

Synthesis of bulk GaSe crystals. The synthesis was carried out in a tube furnace system equipped with a 2" quartz tube. $\mathrm{Ga}_{2} \mathrm{Se}_{3}$ (99.99\%, Alfa Aesar) and $\mathrm{Ga}(99.99 \%$, Alfa Aesar) powders were mixed at a molar ratio of $1: 1$ and place at the center of the quartz tube. The tube was sealed and evacuated to $\sim 5 \times 10^{-3}$ Torr. The furnace was then heated to $950^{\circ} \mathrm{C}$ (from 25 to $700^{\circ} \mathrm{C}$ in $35 \mathrm{~min}$ and 700 to $950^{\circ} \mathrm{C}$ in $25 \mathrm{~min}$ ) and maintained at temperature for $30 \mathrm{~min}$. During synthesis, argon gas was used as a protective gas at $50 \mathrm{sccm}$, and the pressure was maintained at 300 Torr. Following growth, the furnace was cooled naturally to room temperature.

Growth of 2D GaSe crystals. The synthesis of 2D GaSe was carried out in a tube furnace system equipped with a 1" quartz tube (Figure S1). Bulk GaSe crystals and $\mathrm{Ga}_{2} \mathrm{Se}_{3}$ powder were mixed together $\left(\mathrm{GaSe}: \mathrm{Ga}_{2} \mathrm{Se}_{3}\right.$ molar ratio $\left.\sim 50 / 1\right)$, and were used as source materials. $\mathrm{SiO}_{2}(\sim 300 \mathrm{~nm}) / \mathrm{Si}$ pieces $\left(1 \times 1 \mathrm{~cm}^{2}\right)$ were cleaned with acetone, isopropyl alcohol (IPA), and DI water, and used as growth substrates. In a typical run, $\sim 60 \mathrm{mg}$ of source powder and a piece of $\mathrm{SiO}_{2}(\sim 300 \mathrm{~nm}) / \mathrm{Si}$ substrate were loaded on a quartz boat, and subsequently inserted into the furnace. The source was located at the center of the furnace, with the substrate located $\sim 8-13 \mathrm{~cm}$ downstream. After evacuating the tube to $\sim 5 \times 10^{-3}$ Torr, the reaction was conducted at $750^{\circ} \mathrm{C}$ (with a ramping rate of $20^{\circ} \mathrm{C} / \mathrm{min}$ ) for $2-10 \mathrm{~min}$ at a pressure of 30 Torr and an argon carrier gas flow rate of 50-100 sccm (standard cubic centimeter per minute). The vapor-phase reactants were transported by the flowing argon gas to the growth region, in which the temperature was $\sim 650-720^{\circ} \mathrm{C}$, thereby feeding the growth of the $2 \mathrm{D}$ GaSe crystals. After growth, the furnace was cooled naturally to room temperature.

Fabrication of 2D GaSe devices. Electron beam lithography (FEI DB-FIB with Raith pattern writing software) was used for 2D GaSe FET fabrication. First, a layer of PMMA 495A4 was spun-coat on the $2 \mathrm{D}$ GaSe crystals, followed by a $180^{\circ} \mathrm{C}$ bake. After pattern writing and development, a $5 \mathrm{~nm}$ layer of Ti followed by a $30 \mathrm{~nm}$ layer of Au was deposited using electron beam evaporation. Finally, well-defined source and drain electrodes were revealed using lift-off process with Acetone/IPA. 
Characterization and theoretical calculation. The morphologies of the $2 \mathrm{D}$ GaSe crystals were characterized using optical microscopy (Leica DM4500 P), scanning electron microscopy (SEM; Zeiss Merlin SEM), and atomic force microscopy (AFM; Bruker Dimension Icon AFM). The composition was analyzed using energydispersive $\mathrm{x}$-ray spectroscopy (EDS) within the SEM.

The crystal structures of the 2D GaSe were investigated using transmission electron microscopy (TEM), dark-field TEM (DF-TEM), and aberration-corrected scanning transmission electron microscopy (ADF-STEM). TEM imaging and diffraction were conducted using an FEI Technai T12 at $100 \mathrm{kV}$ at low dose densities, and no detectable damage was observed during imaging. Acquisition times for DF-TEM images (both displaced-aperture and centered DF-TEM) were $2 \mathrm{~s}$ per frame. The ADF-STEM image was obtained using an aberration-corrected Nion UltraSTEM operating at $60 \mathrm{kV}$, using a half-angle range of the ADF detector from 86 to $200 \mathrm{mrad}$. The samples for TEM and STEM analysis were grown directly on amorphous silicon films ( $5 \mathrm{~nm}$ in thickness) supported by silicon TEM grid using the same growth process as described above.

Raman measurements were performed using $\mathrm{cw}$ excitation at $532 \mathrm{~nm}$ under a microscope (Jobin Yvon Horiba, T6400) using a long distance objective (100×, N/A $=0.8$ ). The spot size on the sample was $\sim 1 \mu \mathrm{m}$. During Raman mapping, the laser energy at the sample was maintained at $\sim 1 \mathrm{~mW}$, and the acquisition time was minimized to $\sim 1 \mathrm{~s}$ for each $1 \mu \mathrm{m}$ step to prevent photo-degradation of the sample.

The electrical properties and photoresponse of $2 \mathrm{D}$ GaSe devices were measured in vacuum under a probe station using a semiconductor analyzer (Keithley 4200) and a laser driven white light source. The dark state was measured without white light illumination, and it showed p-type semiconducting behavior when the gate voltage was swept at $\mathrm{V}_{\mathrm{ds}}=-10 \mathrm{~V}$. The power intensity of white light source was measured to be $1.2 \mathrm{~mW} / \mathrm{cm}^{2}$ from 400 to $800 \mathrm{~nm}$, and it was used for both the electrical and photoresponse measurements.

The electronic band structures of 2D GaSe crystals were investigated using a highly accurate, all-electron first-principles quantum mechanical calculation code (FHIaims). The exchange-correlation potential of the Perdew-Burke-Ernzerhof (PBE) version of the generalized-gradient approximation (GGA) was used. For k-point samplings, $11 \times 11 \times 6$ mesh points were used for bulk and $11 \times 11 \times 1$ for the films with a large size vacuum $(\sim 50 \AA)$. Different phases of bulk GaSe structures were modeled, such as $\varepsilon, \beta, \delta$, and rhombohedral phases using experimental lattice parameters. All the bulk phases except the rhombohedral structure essentially have very similar electronic features such as bandgap energy, where valance band maximum (VBM) and conduction band minimum (CBM) are located at the $\mathrm{G}$ point. On the other hand, the rhombohedral structure shows an indirect bandgap with significantly larger bandgap energy to other phases by $\sim 1 \mathrm{eV}$. Considering its large bandgap, we believe that the rhombohedral structure is not the observed structure in our experiments. We modeled multi-layered configurations of $\varepsilon$-GaSe to systematically study the changes in the electronic properties in terms of layer numbers.

1. Novoselov, K. S. et al. Two-dimensional atomic crystals. Proc. Natl. Acad. Sci. U.S.A. 102, 10451-10453 (2005).

2. Geim, A. K. \& Novoselov, K. S. The rise of graphene. Nat. Mater. 6, 183-191 (2007)

3. Xu, M. S., Liang, T., Shi, M. M. \& Chen, H. Z. Graphene-like two-dimensional materials. Chem. Rev. 113, 3766-3798 (2013).

4. Bulter, S. Z. et al. Progress, challenges, and opportunities in two-dimensional materials beyond graphene. ACS Nano 7, 2898-2926 (2013).

5. Novoselov, K. S. et al. Electric field effect in atomically thin carbon films. Science 306, 666-669 (2004).

6. Schwierz, F. Graphene transistors. Nat. Nanotechnol. 5, 487-496 (2010).

7. Novoselov, K. S. et al. A roadmap for graphene. Nature 490, 192-200 (2012).

8. Chhowalla, M. et al. The chemistry of two-dimensional layered transition metal dichalcogenide nanosheets. Nat. Chem. 5, 263-275 (2013).

9. Coleman, J. N. et al. Two-dimensional nanosheets produced by liquid exfoliation of layered materials. Science 331, 568-571 (2011).

10. Song, L. et al. Large scale growth and characterization of atomic hexagonal boron nitride layers. Nano Lett. 10, 3209-3215 (2010).

11. Radisavljevic, B., Radenovic, A., Brivio, J., Giacometti, V. \& Kis, A. Single-layer $\mathrm{MoS}_{2}$ transistors. Nat. Nanotechnol. 6, 147-150 (2011).

12. Lee, Y.-H. et al. Synthesis of large-area $\mathrm{MoS}_{2}$ atomic layers with chemical vapor deposition. Adv. Mater. 24, 2320-2325 (2012).

13. van der Zande, A. M. et al. Grains and grain boundaries in highly crystalline monolayer molybdenum disulphide. Nat. Mater. 12, 554-561 (2013).

14. Najmaei, S. et al. Vapour-phase growth and grain boundary structure of molybdenum disulphide atomic layers. Nat. Mater. 12, 754-759 (2013).

15. Lee, Y.-H. et al. Synthesis and transfer of single-layer transition metal disulfides on diverse surface. Nano Lett. 13, 1852-1857 (2013).

16. Zhang, Y. et al. Controlled growth of high-quality monolayer $\mathrm{WS}_{2}$ layers on sapphire and imaging its grain boundary. ACS Nano 7, 8963-8971 (2013).

17. Jones, A. M. et al. Optical generation of excitonic valley coherence in monolayer $\mathrm{WSe}_{2}$. Nat. Nanotechnol. 8, 634-638 (2013).

18. Fang, H. et al. High-performance single layered $\mathrm{WSe}_{2} \mathrm{p}-\mathrm{FET}$ s with chemically doped contacts. Nano Lett. 12, 3788-3792 (2012).

19. Mak, K. F., Lee, C., Hone, J., Shan, J. \& Heinz, T. F. Atomically thin $\mathrm{MoS}_{2}$ : A new direct-gap semiconductor. Phys. Rev. Lett. 105, 136805 (2010).
20. Zeng, H. L., Dai, J. F., Yao, W., Xiao, D. \& Cui, X. D. Valley polarization in $\mathrm{MoS}_{2}$ monolayers by optical pumping. Nat. Nanotechnol. 7, 490-493 (2012).

21. Hu, P. A. et al. Highly responsive ultrathin $\mathrm{GaS}$ nanosheet photodetectors on rigid and flexible substrates. Nano Lett. 13, 1649-1654 (2013).

22. Late, D. J. et al. GaS and GaSe ultrathin layer transistors. Adv. Mater. 24 3549-3554 (2012).

23. Hu, P. A., Wen, Z. Z., Wang, L. F., Tan, P. H. \& Xiao, K. Synthesis of few-layer GaSe nanosheets for high performance photodetectors. ACS Nano 6, 5988-5994 (2012).

24. Lei, S. D. et al. Synthesis and photoresponse of large GaSe atomic layers. Nano Lett. 13, 2777-2781 (2013).

25. Mudd, G. W. et al. Tuning the bandgap of exfoliated InSe nanosheets by quantum confinement. Adv. Mater. 25, 5714-5718 (2013).

26. Ghalouci, L. et al. First principle investigation into hexagonal and cubic structures of gallium selenide. Comput. Mater. Sci. 67, 73-82 (2013).

27. Le Toullec, R., Balkanski, M., Besson, J. M. \& Kuhn, A. Optical absorption edge of a new GaSe polytype. Phys. Lett. A 55, 245-246 (1975).

28. Capozzi, V. \& Montagna, M. Opctial spectroscopy of extrinsic recombinations in gallium selenide. Phys. Rev. B 40, 3182-3190 (1989).

29. Kuhn, A., Chevy, A. \& Chevalier, R. Crystal structure and interatomic distances in GaSe. Phys. Stat. Sol. (a) 31, 469-475 (1975).

30. Plucinski, L. et al. Electronic band structure of GaSe(0001): Angle-resolved photoemission and ab initio theory. Phys. Rev. B 68, 125304 (2003).

31. Augelli, V., Manfredotti, C., Murri, R. \& Vasanelli, L. Hall-mobility anisotropy in GaSe. Phys. Rev. B 17, 3221-3226 (1978).

32. Belen'kii, G. L., Godzhaev, M. O., Salaev, E. Y. \& Aliev, E. T. High-temperature electron-hole liquid in layered InSe, GaSe and GaS crystals. Sov. Phys. JETP 64, 1117-1123 (1986).

33. Fan, Y., Bauer, M., Kador, L., Allakhverdiev, K. R. \& Salaev, E. Y. Photoluminescence frequency up-conversion in GaSe single crystals as studied by confocal microscopy. J. Appl. Phys. 91, 1081-1086 (2002).

34. Leontie, L., Evtodiev, I., Nedeff, V., Stamate, M. \& Caraman, M. Photoelectric properties of $\mathrm{Bi}_{2} \mathrm{O}_{3} / \mathrm{GaSe}$ heterojunctions. Appl. Phys. Lett. 94, 071903 (2009)

35. Shi, W., Ding, Y. J., Fernelius, N. \& Vodopyanov, K. Efficient, tunable, and coherent $0.18-5.27-\mathrm{THz}$ source based on GaSe crystal. Opt. Lett. 27, 1454 (2002).

36. Allakhverdiev, K. R, Yetis, M. O, Ozbek, S, Baykara, T. K. \& Salaev, E. Y. Effective nonlinear GaSe crystal. Optical properties and applications. Laser Phys. 19, 1092-1104 (2009).

37. Rybkovskiy, D. V. et al. Size-induced effects in gallium selenide electric structure: The influence of interlayer interaction. Phys. Rev. B 84, 085314 (2011).

38. Ma, Y. D., Dai, Y., Guo, M., Yu, L. \& Huang, B. B. Tunable electronic and dielectric behavior of GaS and GaSe monolayers. Phys. Chem. Chem. Phys. 15, 7098-7105 (2013).

39. Zólyomi, V., Drummond, N. D. \& Fal'ko, V. I. Band structure and optical transitions in atomic layers of hexagonal gallium chalcogenides. Phys. Rev. B 87, 195403 (2013).

40. Zhuang, H. L. \& Hennig, R. G. Single-layer group-III monochalcogenides photocatalysts for water splitting. Chem. Mater. 25, 3232-3238 (2013).

41. Li, X. S. et al. Large-area synthesis of high-quality and uniform graphene films on copper foils. Science 324, 1312-1314 (2009).

42. Li, C., Huang, L., Snigdha, G. P., Yu, Y. F. \& Cao, L. Y. Role of boundary layer diffusion in vapor deposition growth of chalcogenide nanosheets: The case of GeS. ACS Nano 6, 8868-8877 (2012).

43. Rumaner, L. E., Gray, J. L. \& Ohuchi, F. S. Nucleation and growth of GaSe on GaAs by van der Waal epitaxy. J. Cryst. Growth 177, 17-27 (1997).

44. Ludviksson, A., Rumaner, L. E., Rogers Jr, J. W. \& Ohuchi, F. S. Vacuum sublimation of GaSe: a molecular source for deposition of GaSe. J. Cryst. Growth 151, 114-120 (1995).

45. Eaglesham, D. J. \& Cerullo, M. Dislocation-free Stranski-Krastanow growth of Ge on Si(100). Phys. Rev. Lett. 64, 1943-1946 (1990).

46. Allakhverdiev, K. et al. Lattice vibrations of pure and doped GaSe. Mater. Res. Bull. 41, 751-763 (2006)

47. Late, D. J., Liu, B., Matte, H. S. S. R., Rao, C. N. R. \& Dravid, V. P. Rapid characterization of ultrathin layers of chalcogenides on $\mathrm{SiO}_{2} / \mathrm{Si}$ substrates. $A d v$. Funct. Mater. 22, 1894-1905 (2012).

48. Blum, V. et al. Ab initio molecular simulations with numeric atom-centered orbitals. Comput. Phys. Comm. 180, 2175-2196 (2009).

49. Perdew, J. P., Burke, K. \& Ernzerhof, M. Generalized gradient approximation made simple. Phys. Rev. Lett. 77, 3865-3868 (1996).

50. Nagell, S., Baldereschi, A. \& Maschke, K. Tight-binding study of the electronic states in GaSe polytypes. J. Phys. C: Solid State Phys. 12, 1625-1639 (1979).

51. Lei, S. D. et al. Evolution of the electronic band structures and efficient photodetection in atomic layers of InSe. ACS Nano DOI: 10.1021/nn405529r.

\section{Acknowledgments}

Growth, synthesis, and theoretical studies sponsored by the Laboratory Directed Research and Development (LDRD) program at Oak Ridge National Laboratory. Materials and device characterization conducted at the Center for Nanophase Materials Sciences, which is sponsored at Oak Ridge National Laboratory by the Scientific User Facilities Division, Office of Basic Energy Sciences, U.S. Department of Energy. Synthesis science supported by 
the Materials Science and Energy Division, Office of Basic Energy Sciences, U.S. Department of Energy. Computing resources provided by the National Energy Research Scientific Computing Center, which is supported by the Office of Science of the U.S. Department of Energy under Contract No. DE-AC02-05CH11231.

\section{Author contributions}

K.X., X.F.L., C.M.R. and D.B.G. designed the experiments. X.F.L. synthesized the materials and performed optical microscopy, SEM, AFM characterizations. A.A.P. developed growth mechanisms and performed micro-Raman and PL spectroscopy measurements. J.C.I. performed the ADF-STEM characterization. M.F.C. and M.C. performed TEM and DF-TEM characterization. M.W.L. and I.I.K. fabricated the device and performed electrical and photoresponse measurements. M.Y. performed theory and modeling. X.F.L. and K.X. wrote the manuscript. All authors contributed to the discussion and final manuscript.

\section{Additional information}

Supplementary information accompanies this paper at http://www.nature.com/ scientificreports

Competing financial interests: The authors declare no competing financial interests. How to cite this article: Li, X. et al. Controlled Vapor Phase Growth of Single Crystalline, Two-Dimensional GaSe Crystals with High Photoresponse. Sci. Rep. 4, 5497; DOI:10.1038/ srep05497 (2014).

This work is licensed under a Creative Commons Attribution 4.0 International License. The images or other third party material in this article are included in the article's Creative Commons license, unless indicated otherwise in the credit line; if the material is not included under the Creative Commons license, users will need to obtain permission from the license holder in order to reproduce the material. To view a copy of this license, visit http://creativecommons.org/licenses/by/4.0/ 